HB31

M415

no. $06-25$

2006 


\section{Digitized by the Internet Archive in 2011 with funding from Boston Library Consortium Member Libraries}




Massachusetts Institute of Technology Department of Economics Working Paper Series

\title{
PARTIALLY OPTIMAL ROUTING
}

\author{
Daron Acemoglü \\ Ramesh Johari \\ Asuman Ozdaglar
}

\author{
Working Paper 06-25 \\ May 22, 2006 \\ Room E52-251 \\ 50 Memorial Drive \\ Cambridge, MA 02142
}

This paper can be downloaded without charge from the Social Science Research Network Paper Collection at http://ssrn.com/abstract $=924688$ 


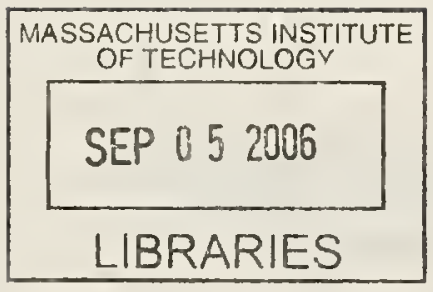




\title{
Partially Optimal Routing*
}

\section{Daron Acemoglu ${ }^{\dagger}$ Ramesh Johari, ${ }^{\ddagger}$ and Asuman Ozdaglar ${ }^{\S}$}

May 22, 2006

\begin{abstract}
Most large-scale communication networks, such as the Internet, consist of interconnected administrative domains. While source (or selfish) routing, where transmission follows the least cost path for each source, is reasonable across domains, service providers typically engage in traffic engineering to improve operating performance within their own network. Motivated by this observation, we develop and analyze a model of partially optimal routing, where optimal routing within subnetworks is overlaid with selfish routing across domains. We demonstrate that optimal routing within a subnetwork does not necessarily improve the performance of the overall network. In particular, when Braess' paradox occurs in the network, partially optimal routing may lead to worse overall network performance. We provide bounds on the worst-case loss of efficiency that can occur due to partially optimal routing. For example, when all congestion costs can be represented by affine latency functions and all administrative domains have a single entry and exit point, the worst-case loss of efficiency is no worse than $25 \%$ relative to the optimal solution. In the presence of administrative domains incorporating multiple entry and/or exit points, however, the performance of partially optimaI routing can be arbitrarily inefficient even with linear latencies. We also provide conditions for traffic engineering to be individually optimal for service providers.
\end{abstract}

${ }^{*}$ This work was partially supported by research grants from the Okawa Foundation and the National Science Foundation. We thank Nicolas Stier-Moses for helpful discussions on the proof of Lemma 4.

tDepartment of Economics, Massachusetts Institute of Technology, Cambridge, MA, 02139 (e-mail: daron@mit.edu).

${ }^{\ddagger}$ Department of Management Science and Engineering, Stanford University, Stanford, CA, 94305 (e-mail: ramesh.johari@stanford.edu).

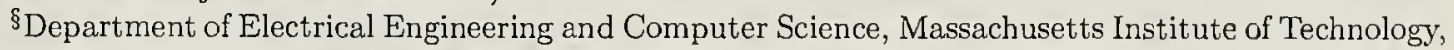
Cambridge, MA, 02139 (e-mail: asuman@mit.edu). 


\section{Introduction}

Since the passage of the Telecommunications Act in 1996, the Internet has undergone a dramatic transformation and experienced increasing decentralization. Today, thousands of network providers cooperate and compete to provide end-to-end network service to billions of users worldwide. While end-users care about the performance across the entire network, individual network providers optimize their own objectives. The Internet's architecture provides no guarantees that provider incentives will be aligned with enduser objectives.

The emergence of overlay routing over the past five years has further highlighted the potentially conflicting objectives of the service provider and the end-users. In overlay routing, end-user software (such as peer-to-peer file-sharing software) makes route selection decisions on the basis of the best end-to-end performance available at any given time, while administrative domains control the routing of traffic within their own (sub)networks. Network operators use traffic engineering to optimize performance, and also to react to the global routing decisions of overlay networks (e.g., [1]).

These considerations make it clear that the study of routing patterns and performance in large-scale communication networks requires an analysis of partially optimal routing, where end-to-end route selection is selfish and responds to aggregate route latency, but network providers redirect traffic within their own networks to achieve minimum intradomain total latency.

We develop and analyze a model of partially optimal routing, combining selfish across-domain routing and traffic engineering by service providers within their administrative domains. While recent research (e.g., $[2,3,4,5,6]$ ) has studied the interactions of overlay routing and traffic engineering, it has neither provided a model of partially optimal routing nor addressed the central question of whether partially optimal routing improves overall network performance.

We consider routing flows between multiple source-destination pairs through a network. Each link is endowed by a latency function describing the congestion level (e.g., delay or probability of packet loss) as a function of the total flow passing through the link (e.g., [7]). Each source-destination pair in the network has a fixed amount of flow, and flows follow the minimum delay route among the available paths as captured by the familiar notion of Wardrop equilibrium (e.g., [7]). Our innovation is to allow subsets of the links in the network ("subnetworks") to be independently owned and operated by different providers, and consider the possibility that these providers engage in traffic engineering and route traffic to minimize the total (or average) latency within their subnetworks. Source-destination pairs sending traffic across subnetworks perceive only the effective latency resulting from the traffic engineering of the service providers. The resulting equilibrium, which we call a partially optimal routing ( $\mathrm{POR}$ ) equilibrium, is a Wardrop equilibrium according to the effective latencies seen by the source-destination pairs. This model provides a stylized description of the practice of traffic engineering in 
the Internet.

Because of the congestion externalities created by selfish routing, the Wardrop equilibrium without traffic engineering within subnetworks is typically inefficient and leads to a level of total delay in excess of the system optimum (see, for example, [8, 9, 10]). It may therefore be conjectured that the addition of traffic engineering within parts of the network will improve performance. Our first set of results show that this is not necessarily the case. In particular, when the Braess' paradox occurs within the global network, partially optimal routing may be less efficient than the Wardrop equilibrium. ${ }^{1}$

Motivated by this finding, we study the extent of inefficiency of partially optimal routing relative to the system optimum. For the case in which all independently-operated subnetworks have unique entry and exit points, and latency functions belong to certain subclasses, we provide tight bounds for the inefficiency of partially optimal routing that exactly match the corresponding bounds for the performance of Wardrop equilibria. For example, with affine latency functions, the worst-case performance of partially optimal routing is no worse than $25 \%$ relative to the system optimum, matching the same bound provided for Wardrop equilibrium by Roughgarden and Tardos [9]. Similarly, we provide bounds for the cases in which latency functions are nonnegative polynomials of bounded degree.

Interestingly, however, in the case of subnetworks with multiple entry and exit points, the performance of partially optimal routing can be arbitrarily inefficient even with linear latency functions. This contrasts with the tight bound of $25 \%$ efficiency loss for Wardrop equilibria with linear or affine latency functions (see $[9,10]$ ). In this general case, we can only provide bounds for some special classes of subnetworks with multiple entry and exit points.

We conclude by investigating subnetwork performance measured in terms of total delay (latency) under partially optimal routing. We show that, in the absence of prices per unit of transmission, a service provider may prefer not to engage in traffic engineering in order to reduce total flow and delay in their subnetwork. In addition, we provide conditions for service providers to prefer traffic engineering to selfish routing within their domain.

The remainder of the paper is organized as follows. Section 2 introduces the three basic routing paradigms: socially optimal routing, where total (or average) latency is minimized across the entire network; selfish routing, where end-to-end route selection is made based on minimum route latency; and partially optimal routing, where end-toend route selection is still dependent on aggregate route latency, but providers engage in traffic engineering within their subnetworks to achieve minimum intradomain total latency.

Section 3 analyzes the performance of partially optimal routing. We show that there may exist situations where optimization within a subnetwork leads to lower global

\footnotetext{
${ }^{1}$ Throughout, by Wardrop equilibrium we refer to the equilibrium of the same network structure without any traffic engineering-without any optimal routing within subnetworks.
} 
network performance. We prove that this can only be the case when the Braess' paradox occurs within the global network. Section 4 then analyzes the worst-case efficiency loss that can occur at the partially optimal routing solution and establishes bounds on efficiency loss when all latency functions are affine, and when all latency functions are nonnegative polynomials of bounded degree. In the special case where all latency functions are affine, we find that the ratio of partially optimal routing cost to the social optimum is no worse than $4 / 3$.

In Section 5, we consider the case where subnetworks may have multiple entry and exit points, and show how partially optimal routing leads to further inefficiencies in this case. Section 6 considers the choice of routing policy by a single service provider and provides conditions under which traffic engineering is (individually) optimal for a provider in parallel link topologies. We conclude in Section 7.

\section{Preliminaries: Different Routing Paradigms}

We consider a directed network $G=(V, A)$, with node set $V$, link (or edge) set $A$, and $w$ source-destination node pairs $\left\{s_{1}, t_{1}\right\}, \ldots,\left\{s_{w}, t_{w}\right\}$. Let $W=\{1, \ldots, w\}$. Let $P_{i}$ denote the set of paths available from $s_{i}$ to $t_{i}$ using the edges in $A$; we view each path $p \in P_{i}$ as a subset of $A, p \subset A$. Define $P=\cup_{i \in W} P_{i}$. Each link $j \in A$ has a strictly increasing, nonnegative latency function $l_{j}\left(x_{j}\right)$ as a function of the flow on link $j{ }^{2}$ We assume that $X_{i}$ units of flow are to be routed from $s_{i}$ to $t_{i}$, for all $i \in W$, and we define $\mathrm{X}=\left[X_{1}, \ldots, X_{w}\right]$. We call the tuple $R=(V, A, P, s, t, \mathrm{X}, \mathrm{l})$ a routing instance. We denote the set of routing instances $R=(V, A, P, s, t, \mathrm{X}, \mathrm{l})$ by the set $\mathcal{R}$. In the following, we will also be interested in routing instances in which the latency functions of all links are restricted to belong to a certain class of functions. We denote the set of routing instances $R$ in which all latency functions are convex (affine and concave, respectively) by $\mathcal{R}^{\text {conv }}$ ( $\mathcal{R}^{a f f}$ and $\mathcal{R}^{\text {conc }}$, respectively).

\footnotetext{
${ }^{2}$ Throughout the paper, we will refer to $l_{j}$ as the latency function, though $l_{j}$ can be used to model congestion metrics other than latency (e.g., loss).
} 


\subsection{Socially Optimal Routing}

Given a routing instance $R=(V, A, P, s, t, \mathrm{X}, \mathrm{l})$, we define the social optimum, denoted by $\mathrm{x}^{S O}(R)$, as the optimal solution of the following optimization problem:

$$
\begin{aligned}
\operatorname{minimize} & \sum_{j \in A} x_{j} l_{j}\left(x_{j}\right) \\
\text { subject to } & \sum_{p \in P: j \in p} y_{p}=x_{j}, \quad j \in A \\
& \sum_{p \in P_{i}} y_{p}=X_{i}, \quad i \in W \\
& y_{p} \geq 0, \quad p \in P .
\end{aligned}
$$

This optimization problem minimizes the total (or equivalently the average) delay experienced over all paths. Under our assumption that each latency function is continuous, it follows that $\mathrm{x}^{S O}(R)$ is well-defined. The total latency cost at the social optimum is given by:

$$
C\left(\mathrm{x}^{S O}(R)\right)=\sum_{j \in A} x_{j}^{S O}(R) l_{j}\left(x_{j}^{S O}(R)\right)
$$

\subsection{Selfish Routing}

When traffic routes "selfishly" - that is, when sources choose minimum delay end-to-end paths-all paths with nonzero flow must have the same total delay. A flow configuration with this property is called a Wardrop equilibrium. Under the assumptions on the latency functions (i.e., each $l_{j}$ is continuous and strictly increasing), it is well-known that the Wardrop equilibrium flow vector for a given routing instance $R$, denoted $x^{W E}(R)$, is the unique optimal solution to the following optimization problem (see e.g., $[9,11]$ ):

$$
\begin{aligned}
\text { minimize } & \sum_{j \in A} \int_{0}^{x_{j}} l_{j}(z) d z \\
\text { subject to } & \sum_{p \in P: j \in p} y_{p}=x_{j}, \quad j \in A ; \\
& \sum_{p \in P_{i}} y_{p}=X_{i}, \quad i \in W ; \\
& y_{p} \geq 0, \quad p \in P .
\end{aligned}
$$

The total latency cost at the Wardrop equilibrium is given by

$$
C\left(\mathrm{x}^{W E}(R)\right)=\sum_{j \in A} x_{j}^{W E}(R) l_{j}\left(x_{j}^{W E}(R)\right) .
$$


Equivalently a feasible solution $\mathrm{x}^{W E}$ for a routing instance $R$ is a Wardrop equilibrium if and only if it satisfies

$$
\sum_{j \in A} l_{j}\left(x_{j}^{W E}\right)\left(x_{j}^{W E}-x_{j}\right) \leq 0,
$$

for all feasible solutions $\mathrm{x}$ for the same routing instance; see, for example, $[12,13]$.

\subsection{Partially Optimal Routing}

Let us now assume that a single network provider controls a subnetwork with unique entry and exit points; within this domain, the provider optimizes performance of traffic flow. Formally, we assume there is a collection of directed subgraphs (subnetworks) inside of $G$. Within a subnetwork $G_{0}=\left(V_{0}, A_{0}\right)$, a service provider optimally routes all incoming traffic. Let $s_{0} \in V_{0}$ denote the unique entry point to $G_{0}$, and let $t_{0} \in V_{0}$ denote the unique exit point from $G_{0}$. Let $P_{0}$ denote the set of available paths from $s_{0}$ to $t_{0}$ using the edges in $A_{0}$. We make the assumption that every path in $P$ passing through any node in $V_{0}$ must contain a path in $P_{0}$ from $s_{0}$ to $t_{0}$; this is consistent with our assumption that $G_{0}$ is an independent autonomous system, with a unique entry and exit point. We call $R_{0}=\left(V_{0}, A_{0}, P_{0}, s_{0}, t_{0}\right)$ a subnetwork of $G$, and with a slight abuse of notation, we say that $R_{0} \subset R$.

Given an incoming amount of flow $X_{0}$, the network provider chooses a routing of flow to solve the following optimization problem to minimize total (or average) latency:

$$
\begin{aligned}
\text { minimize } & \sum_{j \in A_{0}} x_{j} l_{j}\left(x_{j}\right) \\
\text { subject to } & \sum_{p \in P_{0}: j \in p} y_{p}=x_{j}, \quad j \in A_{0} ; \\
& \sum_{p \in P_{0}} y_{p}=X_{0} ; \\
& y_{p} \geq 0, \quad p \in P_{0} .
\end{aligned}
$$

In this optimization problem, the subnetwork owner sees an incoming traffic amount $X_{0}$, and chooses the optimal routing of this flow through the subnetwork. This is a formal abstraction of the process of traffic engineering carried out by many network providers to optimize intradomain performance.

Let $L\left(X_{0}\right)$ denote the optimal value of the preceding optimization problem. We define $l_{0}\left(X_{0}\right)=L\left(X_{0}\right) / X_{0}$ as the effective latency of partially optimal routing in the subnetwork $R_{0}$, with flow $X_{0}>0$. If traffic in the entire network $G$ routes selfishly, while traffic is optimally routed within $G_{0}$, then replacing $G_{0}$ by a single link with latency $l_{0}$ will leave the Wardrop equilibrium flow unchanged elsewhere in $G$.

We have the following simple lemma that provides basic properties of $l_{0}$ and $L$. 
Lemma 1 Assume that every latency function, $l_{j}$, is a strictly increasing, nonnegative, and continuous function. Then:

(a) The effective latency $l_{0}\left(X_{0}\right)$ is a strictly increasing function of $X_{0}>0$.

(b) Assume further that each $l_{j}$ is a convex function. The total cost $L\left(X_{0}\right)$ is a convex function of $X_{0}$.

Proof.

(a) Fix $X_{0}$, and $\hat{X}_{0}<X_{0}$ with $\hat{X}_{0}>0$. Let $(\mathrm{x}, \mathrm{y})$ be an optimal solution to problem (4) with total flow $X_{0}$. Define $\hat{y}_{p}=y_{p} \hat{X}_{0} / X_{0}$, for all $p \in P_{0}$. Then this is a feasible solution to problem (4) with total flow $\hat{X}_{0}$. Furthermore, the total latency at this solution is easily seen to be:

$$
\sum_{p \in P_{0}} \hat{y}_{p} \sum_{j \in p} l_{j}\left(\sum_{q \in P_{0}: j \in q} \hat{y}_{q}\right)=\sum_{j \in A_{0}} \frac{x_{j} \hat{X}_{0}}{X_{0}} l_{j}\left(\frac{x_{j} \hat{X}_{0}}{X_{0}}\right) .
$$

Now we observe that:

$$
\begin{aligned}
l_{0}\left(\hat{X}_{0}\right) & =\sum_{j \in A_{0}} \frac{x_{j}}{X_{0}} l_{j}\left(\frac{x_{j} \hat{X}_{0}}{X_{0}}\right) \\
& <\frac{1}{X_{0}} \sum_{j \in A_{0}} x_{j} l_{j}\left(x_{j}\right)=l_{0}\left(X_{0}\right),
\end{aligned}
$$

where the last inequality follows because $l_{j}$ is strictly increasing and $\hat{X}_{0}<X_{0}$.

(b) Note that $L\left(X_{0}\right)$ is the primal function of optimization problem (4). Since the objective function is convex and the constraints are linear, the result follows using standard arguments from convex analysis (see [14]).

In light of the preceding lemma, we can extend the definition of $l_{0}$ so that $l_{0}(0)=$ $\lim _{x_{0} \downarrow 0} l_{0}\left(x_{0}\right)$; the preceding limit is well defined since $l_{0}$ is strictly increasing.

To define the overall network performance under partially optimal routing, first suppose that there is a single independently-operated subnetwork. Given a routing instance $R=(V, A, P, s, t, \mathrm{X}, \mathrm{l})$, and a subnetwork $R_{0}=\left(V_{0}, A_{0}, P_{0}, s_{0}, t_{0}\right)$ defined as above, we define a new routing instance $R^{\prime}=\left(V^{\prime}, A^{\prime}, P^{\prime}, s, t, \mathrm{X}, \mathrm{I}^{\prime}\right)$ as follows:

$$
\begin{aligned}
& V^{\prime}=\left(V \backslash V_{0}\right) \bigcup\left\{s_{0}, t_{0}\right\} ; \\
& A^{\prime}=\left(A \backslash A_{0}\right) \bigcup\left\{\left(s_{0}, t_{0}\right)\right\} ;
\end{aligned}
$$


$P^{\prime}$ corresponds to all paths in $P$, where any subpath in $P_{0}$ is replaced by the link $\left(s_{0}, t_{0}\right)$; and $\mathbf{l}^{\prime}$ consists of latency functions $l_{j}$ for all edges in $A \backslash A_{0}$, and latency $l_{0}$ for the edge $\left(s_{0}, t_{0}\right)$. Thus $R^{\prime}$ is the routing instance $R$ with the subgraph $G_{0}$ replaced by a single link with latency $l_{0}$; we call $R^{\prime}$ the equivalent $P O R$ instance for $R$ with respect to $R_{0}$. The overall network flow in $R$ with partially optimal routing in $R_{0}, \mathrm{x}^{P O R}\left(R, R_{0}\right)$, is defined to be the Wardrop equilibrium flow in the routing instance $R^{\prime}$ :

$$
\mathrm{x}^{P O R}\left(R, R_{0}\right)=\mathrm{x}^{W E}\left(R^{\prime}\right) .
$$

In other words, it is equilibrium with traffic routed selfishly given the effective latency $l_{0}$ of the subnetwork $R_{0}$. Note also that this formulation leaves undefined the exact flow in the subnetwork $R_{0}$; this is to be expected, since problem (4) may not have a unique solution.

The total latency cost of the equivalent POR instance for $R$ with respect to $R_{0}$ is given by

$$
C\left(\mathbf{x}^{P O R}\left(R, R_{0}\right)\right)=\sum_{j \in A^{\prime}} x_{j}^{P O R}\left(R, R_{0}\right) l_{j}\left(x_{j}^{P O R}\left(R, R_{0}\right)\right) .
$$

The definition immediately generalizes when there are multiple independently-operated subnetworks. Let $R_{0}^{j}=\left(V_{0}^{j}, A_{0}^{j}, P_{0}^{j}, s_{0}^{j}, t_{0}^{j}\right)$ for $j=1,2, \ldots, J$ denote the subnetworks, each represented by a directed subgraph $G_{0}^{j}$. Define

$$
\begin{aligned}
& V^{\prime}=\left(V \backslash \bigcup_{j=1}^{J} V_{0}^{j}\right) \bigcup_{j=1}^{J}\left\{s_{0}^{j}, t_{0}^{j}\right\} ; \\
& A^{\prime}=\left(A \backslash \bigcup_{j=1}^{J} A_{0}^{j}\right) \bigcup_{j=1}^{J}\left\{\left(s_{0}^{j}, t_{0}^{j}\right)\right\} .
\end{aligned}
$$

Let $R^{\prime}$ be the routing instance $R$ with each subgraph $G_{0}^{j}$ replaced by a single link with effective latency $l_{0}^{j}$. The partially optimal routing flow $\mathrm{x}^{P O R}\left(R,\left\{R_{0}^{j}\right\}_{j=1}^{J}\right)$, is again the Wardrop equilibrium flow in the routing instance $R^{\prime}$. In the remainder of the paper, we assume without loss of generality that there is a single subnetwork in the overall network.

\section{Partially Optimal Routing and Global Performance}

We first consider the effect of optimal routing within subnetworks on the performance of the overall network. One might conjecture that optimally routing traffic within subnetworks should improve the overall performance. The following example shows that this need not be the case. 


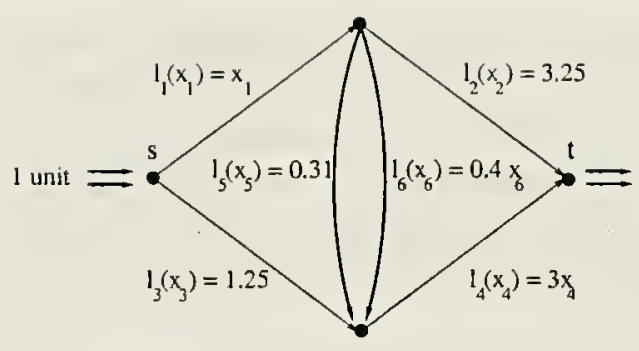

(a)

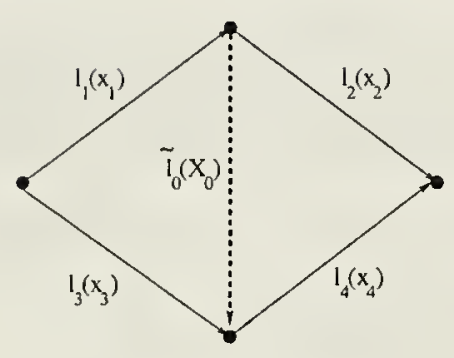

(b)

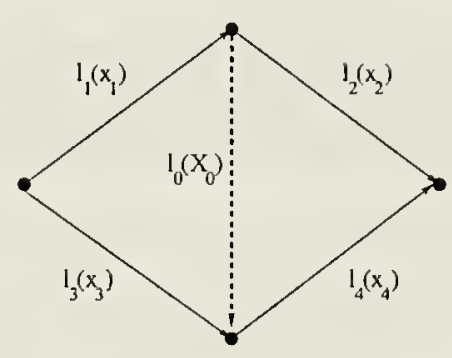

(c)

Figure 1: A network for which POR leads to a worse performance relative to selfish routing. Figures (b) and (c) illustrate representing the subnetwork with a single link with Wardrop effective latency $\tilde{l}_{0}\left(X_{0}\right)$ and optimal effective latency $l_{0}\left(X_{0}\right)$, respectively.

Example 1 Consider the network $G=(V, A)$ with source and destination nodes $s, t \in$ $V$ illustrated in Figure 1(a). Let $R=(V, A, P, s, t, 1, \mathrm{l})$ be the corresponding routing instance, i.e., one unit of flow is to be routed over this network. The subnetwork $G_{0}$ consists of the two parallel links in the middle, links 5 and 6 , with latency functions

$$
l_{5}\left(x_{5}\right)=0.31, \quad l_{6}\left(x_{6}\right)=0.4 x_{6} .
$$

The latency functions for the remaining links in the network are given by

$$
\begin{aligned}
& l_{1}\left(x_{1}\right)=x_{1}, \quad l_{2}\left(x_{2}\right)=3.25, \\
& l_{3}\left(x_{3}\right)=1.25, \quad l_{4}\left(x_{4}\right)=3 x_{4} .
\end{aligned}
$$

Assume first that the flow through the subnetwork $G_{0}$ is routed selfishly, i.e., according to the Wardrop equilibrium. Given a total flow $X_{0}$ through the subnetwork $G_{0}$, the effective Wardrop latency can be defined as

$$
\tilde{l}_{0}\left(X_{0}\right)=\frac{1}{X_{0}} C\left(x^{W E}\left(R_{0}\right)\right)
$$

[cf. Eq. (2)], where $R_{0}$ is the routing instance corresponding to the subnetwork $G_{0}$ with total flow $X_{0}$. The effective Wardrop latency for this example is given by

$$
\tilde{l}_{0}\left(X_{0}\right)=\min \left\{0.31,0.4 X_{0}\right\} \text {. }
$$


Substituting the subnetwork with a single link with latency function $\tilde{l}_{0}$ yields the network in Figure 1(b). It can be seen that selfish routing over the network of Figure 1(b) leads to the link flows $x_{1}^{W E}=0.94$ and $X_{0}^{W E}=0.92$, with a total cost of $C\left(\mathrm{x}^{W E}(R)\right)=4.19$. It is clear that this flow configuration arises from a Wardrop equilibrium in the original network.

Assume next that the flow through the subnetwork $G_{0}$ is routed optimally, i.e., as the optimal solution of problem (4) for the routing instance corresponding to $G_{0}$. Given a total flow $X_{0}$ through the subnetwork $G_{0}$, the effective latency of optimal routing within the subnetwork $G_{0}$ can be defined as

$$
l_{0}\left(X_{0}\right)=\frac{L\left(X_{0}\right)}{X_{0}}
$$

where $L\left(X_{0}\right)$ is the optimal value of problem (4). The effective optimal routing latency for this example is given by

$$
l_{0}\left(X_{0}\right)=\left\{\begin{array}{cc}
0.4 X_{0}, & \text { if } 0 \leq X_{0} \leq 0.3875 \\
0.31-\frac{0.0961}{1.6 X_{0}}, & \text { if } X_{0} \geq 0.3875
\end{array}\right.
$$

Substituting the subnetwork with a single link with latency function $l_{0}$ yields the network in Figure 1(c). Note that selfish routing over this network leads to the partially optimal routing (POR) equilibrium. It can be seen that at the POR equilibrium, the link flows are given by $x_{1}^{P O R}=1$ and $X_{0}^{P O R}=1$, with a total cost of $C\left(\mathrm{x}^{P O R}(R)\right)=4.25$, which is strictly greater than $C\left(\mathrm{x}^{W E}(R)\right)$.

In the preceding example, when the subnetwork optimizes intradomain performance, we see a degradation in global network performance. This is reminiscent of Braess' paradox, a classic example of degradation in global network performance despite local improvements (see, for example, [11]). Braess' paradox occurs in a network if reducing the link latency functions increases the total latency in the network. We now investigate the relationship between Braess' paradox and the performance degradation observed in Example 1.

Definition 1 (Braess' paradox) Consider a routing instance $R=(V, A, P, s, t, \mathbf{X}, \mathrm{l})$ and a subnetwork $R_{0}=\left(V_{0}, A_{0}, P_{0}, s_{0}, t_{0}\right) \subset R$. We say that Braess' paradox occurs in $R$ centered at $R_{0}$ if there exists another routing instance $R_{m}=(V, A, P, s, t, \mathbf{X}, \mathbf{m})$, with a vector of strictly increasing, nonnegative latency functions, $\mathbf{m}=\left(m_{j}, j \in A\right)$, such that for all $x_{j} \geq 0$,

$$
m_{j}\left(x_{j}\right) \leq l_{j}\left(x_{j}\right), \forall j \in A_{0}, \quad m_{j}\left(x_{j}\right)=l_{j}\left(x_{j}\right), \forall j \notin A_{0},
$$

and

$$
C\left(\mathrm{x}^{W E}\left(R_{m}\right)\right)>C\left(\mathrm{x}^{W E}(R)\right) .
$$


In our definition we have explicitly fixed a subnetwork $R_{0}$ within which we locally "improve" performance; formally, the routing instance $R^{\prime}$ differs from $R$ only by a reduction of the latency functions on some (or all) links. Nevertheless, in a network topology where Braess' paradox occurs, this local change can yield a higher total latency.

Similarly, the following definition captures the counterintuitive phenomenon exhibited in Example 1, where traffic engineering within some subnetwork, i.e., partially optimal routing, leads to a degradation in the overall performance compared to pure selfish routing.

Definition 2 (POR paradox) Consider a routing instance $R=(V, A, P, s, t, \mathrm{X}, \mathrm{l})$, and a subnetwork $R_{0}=\left(V_{0}, A_{0}, P_{0}, s_{0}, t_{0}\right)$. We say that the POR paradox (partially optimal routing paradox) occurs in $R$ with respect to $R_{0}$ if

$$
C\left(\mathrm{x}^{P O R}\left(R, R_{0}\right)\right)>C\left(\mathrm{x}^{W E}(R)\right)
$$

Intuitively, the POR paradox appears to be a form of "generalized Braess' paradox", in the following sense. Given a total flow $X_{0}$ routed through the subnetwork $G_{0}$, we define the effective Wardrop latency $\tilde{l}_{0}$, as follows:

$$
\tilde{l}_{0}\left(X_{0}\right)=\frac{1}{X_{0}} \sum_{j \in A_{0}} x_{j}^{W E}\left(R^{\prime}\right) l_{j}\left(x_{j}^{W E}\left(R^{\prime}\right)\right)=\frac{C\left(\mathrm{x}^{W E}\left(R^{\prime}\right)\right)}{X_{0}}
$$

where $R^{\prime}=\left(V_{0}, A_{0}, P_{0}, s_{0}, t_{0}, X_{0}, 1\right)$ is a routing instance corresponding to the subnetwork $R_{0}$ with total flow $X_{0}$ [cf. Eq. (5)]. As in Lemma 1, it is straightforward to show that $\tilde{l}_{0}$ is strictly increasing. Furthermore, it is clear that $\tilde{l}_{0}\left(X_{0}\right) \geq l_{0}\left(X_{0}\right)$ for all $X_{0} \geq 0$, since $\mathrm{x}^{W E}\left(R^{\prime}\right)$ is a feasible solution to problem (4). Thus when we contrast $\mathrm{x}^{P O R}(R)$ and $\mathrm{x}^{W E}(R)$, it is as if we are lowering the effective latency of the subnetwork $R_{0}$. If this increases the total latency, then we are observing a form of Braess' paradox.

ln fact, it is possible to show a stronger result: whenever the POR paradox occurs in $R$ with respect to some $R_{0} \subset R$, then Braess' paradox occurs in $R$ centered at $R_{0}$. This result is stronger than the "generalized Braess' paradox" discussed in the preceding paragraph, because it shows that Braess' paradox occurs within the original instance $R$ without altering the network topology.

Proposition 1 Consider a routing instance $R=(V, A, P, s, t, \mathrm{X}, \mathrm{l})$ and a subnetwork $R_{0}=\left(V_{0}, A_{0}, P_{0}, s_{0}, t_{0}\right) \subset R$. Assume that the POR paradox occurs in $R$ with respect to $R_{0}$. Then Braess' paradox occurs in $R$ centered at $R_{0}$.

Proof. Our approach will be to uniformly lower the latency functions in the subnetwork $R_{0}$, such that we exactly ensure at a Wardrop equilibrium the effective latency of $R_{0}$ is given by $l_{0}$, the effective latency of optimal routing within $R_{0}$. This will allow selfish routing to "replicate" the partially optimal routing of flow, and imply Braess' paradox. 
Let $\mathrm{x}^{W E}(R)$ be the Wardrop equilibrium flow for the routing instance $R$, with corresponding path flows $\mathrm{y}^{W E}(R)$. Similarly, let $\mathrm{x}^{P O R}\left(R, R_{0}\right)$ be the flow with partially optimal routing in $R_{0}$, with corresponding path flows $\mathrm{y}^{P O R}\left(R, R_{0}\right)$. Let $X_{0}=x_{s_{0} t_{0}}^{P O R}\left(R, R_{0}\right)$ represent the flow routed through the subnetwork $R_{0}$ under partially optimal routing. Note that $X_{0}>0$ since by assumption POR paradox occurs in $R$ with respect to $R_{0}$. Let $l_{0}$ denote the effective latency of $R_{0}$ under partially optimal routing, and $\tilde{l}_{0}$ denote the effective latency of $R_{0}$ under selfish routing [cf. Eq. (6)].

Define a routing instance $R_{0}^{\prime}=\left(V_{0}, A_{0}, P_{0}, s_{0}, t_{0}, X_{0}, 1\right)$ and let $\mathrm{x}^{W E}\left(R_{0}^{\prime}\right)$ be the Wardrop equilibrium flow for the routing instance $R_{0}^{\prime}$.

We define a new collection of latency functions as follows. For all $j \notin A_{0}$, define $m_{j}=l_{j}$. For $j \in A_{0}$, we choose a new strictly increasing, nonnegative latency function $m_{j}$ with $m_{j}\left(x_{j}\right) \leq l_{j}\left(x_{j}\right)$ for all $x_{j} \geq 0$; such that

$$
m_{j}\left(x_{j}^{W E}\left(R_{0}^{\prime}\right)\right)=\frac{l_{0}\left(X_{0}\right)}{\tilde{l}_{0}\left(X_{0}\right)} l_{j}\left(x_{j}^{W E}\left(R_{0}^{\prime}\right)\right) .
$$

Observe that such a choice is possible, since $l_{0}\left(X_{0}\right) \leq \tilde{l}_{0}\left(X_{0}\right)$.

Let $T_{0}=\left(V_{0}, A_{0}, P_{0}, s_{0}, t_{0}, X_{0}, \mathbf{m}\right)$; i.e., $T_{0}$ is the routing instance $R_{0}^{\prime}$ with latencies replaced by $\mathrm{m}$. We claim that $\mathrm{x}^{W E}\left(T_{0}\right)=\mathrm{x}^{W E}\left(R_{0}^{\prime}\right)$. This follows from the definition of $\mathrm{m}$ : all values $m_{j}\left(x_{j}^{W E}\left(R_{0}^{\prime}\right)\right)$ are proportional to $l_{j}\left(x_{j}^{W E}\left(R_{0}^{\prime}\right)\right)$, with common constant of proportionality $l_{0}\left(X_{0}\right) / \tilde{l}_{0}\left(X_{0}\right)$. Thus if $\mathrm{x}^{W E}\left(R_{0}^{\prime}\right)$ is the Wardrop equilibrium flow with latencies 1 , it must remain so with latencies $m$. Furthermore, observe that for any path $p$ with positive flow, we have

$$
\sum_{j \in p} m_{j}\left(x_{j}^{W E}\left(T_{0}\right)\right)=\frac{l_{0}\left(X_{0}\right)}{\tilde{l}_{0}\left(X_{0}\right)} \sum_{j \in p} l_{j}\left(x_{j}^{W E}\left(R_{0}^{\prime}\right)\right)=l_{0}\left(X_{0}\right),
$$

because the second summation above is equal to $\tilde{l}_{0}\left(X_{0}\right)$. Thus we conclude

$$
C\left(\mathrm{x}^{W E}\left(T_{0}\right)\right)=\sum_{j \in A_{0}} x_{j}^{W E}\left(T_{0}\right) m_{j}\left(x_{j}^{W E}\left(T_{0}\right)\right)=X_{0} l_{0}\left(X_{0}\right) .
$$

Let $T=(V, A, P, s, t, \mathbf{X}, \mathbf{m})$. Define a feasible flow $\mathbf{x}=\left[x_{j}\right]_{j \in A}$ as follows:

$$
x_{j}= \begin{cases}x_{j}^{P O R}\left(R, R_{0}\right), & \text { if } j \notin A_{0} ; \\ x_{j}^{W E}\left(R_{0}^{\prime}\right), & \text { if } j \in A_{0} .\end{cases}
$$

We claim that $\mathrm{x}^{W E}(T)=\mathbf{x}$. This claim follows easily since we have already established that $\mathrm{x}^{W E}\left(T_{0}\right)=\mathrm{x}^{W E}\left(R_{0}^{\prime}\right)$, and $(7)$ holds. In the flow $\mathrm{x}$ for the routing instance $T$, the effective latency perceived by any flow crossing the subnetwork $R_{0}$ is exactly equal to the partially optimal routing effective latency $l_{0}\left(X_{0}\right)$ (by (7)). But then since all routing outside the subnetwork $R_{0}$ is performed according to $\mathrm{x}^{P O R}\left(R, R_{0}\right)$, we conclude that in fact $\mathrm{x}^{W E}(T)=\mathrm{x}$, as required. 
Combining the preceding, we obtain

$$
\begin{aligned}
\sum_{j \in A} x_{j}^{W E}(T) m_{j}\left(x_{j}^{W E}(T)\right) & \\
= & \sum_{j \notin A_{0}} x_{j}^{P O R}\left(R, R_{0}\right) l_{j}\left(x_{j}^{P O R}\left(R, R_{0}\right)\right) \\
& \quad+\sum_{j \in A_{0}} x_{j}^{W E}\left(R_{0}^{\prime}\right) m_{j}\left(x_{j}^{W E}\left(R_{0}^{\prime}\right)\right) \\
= & \sum_{j \notin A_{0}} x_{j}^{P O R}\left(R, R_{0}\right) l_{j}\left(x_{j}^{P O R}\left(R, R_{0}\right)\right)+X_{0} l_{0}\left(X_{0}\right) \\
= & \sum_{j \in A^{\prime}} x_{j}^{P O R}\left(R, R_{0}\right) l_{j}\left(x_{j}^{P O R}\left(R, R_{0}\right)\right) \\
= & C\left(\mathrm{x}^{P O R}\left(R, R_{0}\right)\right) .
\end{aligned}
$$

Since we assumed that the POR paradox occurs in $R$ with respect to $R_{0}$, we obtain from the preceding that

$$
C\left(\mathrm{x}^{W E}(T)\right)=C\left(\mathrm{x}^{P O R}\left(R, R_{0}\right)\right)>C\left(\mathrm{x}^{W E}(R)\right)
$$

implying that Braess' paradox occurs in $R$-centered at $R_{0}$.

An immediate corollary of the preceding proposition is the following:

Corollary 1 Given a routing instance $R$, if Braess' paradox does not occur in $R$, then partially optimal routing with respect to any subnetwork always improves the network performance.

Since Milchtaich, [15], has shown that Braess' paradox does not occur in graphs with the serial-parallel structure, this corollary implies that as long as the network under consideration has a serial-parallel structure (for example, a network of parallel links), partially optimal routing always improves the overall network performance.

\section{Efficiency of Partially Optimal Routing}

We have seen in Example 1 that partially optimal routing can actually worsen performance relative to the Wardrop equilibrium. In this section, we quantify the inefficiency of partially optimal routing. Our metric of efficiency is the ratio of the total cost at the social optimum to the total cost at the partially optimal routing solution, $C\left(\mathrm{x}^{S O}\right) / C\left(\mathrm{x}^{P O R}\right)$. Throughout, we assume that all independently-operated subnetworks can be represented as subgraphs with unique entry and exit points. 
We will establish two main theorems. The first provides a tight bound on the loss of efficiency when all latency functions are affine; and the second provides a tight bound on the loss of efficiency when all latency functions are polynomials of bounded degree.

We start with a simple result that compares the worst-case efficiency loss of partially optimal routing with that of selfish routing. These relations will be useful in finding tight bounds on the efficiency loss of partially optimal routing. Recall that $\mathcal{R}^{\text {conv }}, \mathcal{R}^{\text {aff }}$, and $\mathcal{R}^{\text {conc }}$ denote the class of all routing instances where latency functions are convex, affine, and concave, respectively.

Proposition 2 (a) For all $\mathcal{R}^{\prime} \in\left\{\mathcal{R}^{\text {conv }}, \mathcal{R}^{\text {aff }}, \mathcal{R}^{\text {conc }}\right\}$, we have

$$
\inf _{\substack{R \in \mathcal{R}^{\prime} \\ R_{0} \subset R}} \frac{C\left(\mathrm{x}^{S O}(R)\right)}{C\left(\mathrm{x}^{P O R}\left(R, R_{0}\right)\right)} \leq \inf _{R \in \mathcal{R}^{\prime}} \frac{C\left(\mathrm{x}^{S O}(R)\right)}{C\left(\mathrm{x}^{W E}(R)\right)} .
$$

(b)

$$
\inf _{\substack{R \in \mathcal{R} \\ R_{0} \subset R}} \frac{C\left(\mathrm{x}^{S O}(R)\right)}{C\left(\mathrm{x}^{P O R}\left(R, R_{0}\right)\right)}=\inf _{R \in \mathcal{R}} \frac{C\left(\mathrm{x}^{S O}(R)\right)}{C\left(\mathrm{x}^{V E}(R)\right)} .
$$

(c)

$$
\inf _{\substack{R \in \mathcal{R}^{a f f} \\ R_{0} \subset R}} \frac{C\left(\mathrm{x}^{S O}(R)\right)}{C\left(\mathrm{x}^{P O R}\left(R, R_{0}\right)\right)} \geq \inf _{\substack{R \in \mathcal{R}^{\text {conc }} \\ \rightarrow}} \frac{C\left(\mathrm{x}^{S O}(R)\right)}{C\left(\mathrm{x}^{W E}(R)\right)}
$$

Proof.

(a) Given an arbitrary routing instance $R=(V, A, P, s, t, \mathrm{X}, \mathrm{l})$, simply let $R_{0}$ consist of a single link $j \in A$ from the routing instance $R$, with the corresponding latency function $l_{j}$. Then it is clear that $\mathrm{x}^{P O R}\left(R, R_{0}\right)=\mathrm{x}^{W E}(R)$; thus for any instance on the right hand side of (8) we have constructed an equivalent instance on the left hand side with the same objective function value, establishing the relation.

(b) The argument in part (a) establishes

$$
\inf _{\substack{R \in \mathcal{R} \\ R_{0} \subset R}} \frac{C\left(\mathrm{x}^{S O}(R)\right)}{C\left(\mathrm{x}^{P O R}\left(R, R_{0}\right)\right)} \leq \inf _{R \in \mathcal{R}} \frac{C\left(\mathrm{x}^{S O}(R)\right)}{C\left(\mathrm{x}^{W E}(R)\right)}
$$

To show the reverse inequality, let $R \in \mathcal{R}$ and $R_{0} \subset \mathcal{R}$. Let $R^{\prime}$ be the equivalent POR instance for $R$ with respect to $R_{0}$. Then it can be seen that

$$
\begin{gathered}
C\left(\mathrm{x}^{P O R}\left(R, R_{0}\right)\right)=C\left(\mathrm{x}^{W E}\left(R^{\prime}\right)\right), \\
C\left(\mathrm{x}^{S O}(R)\right)=C\left(\mathrm{x}^{S O}\left(R^{\prime}\right)\right) .
\end{gathered}
$$

Hence, for every feasible solution of the optimization problem on the left-hand side of relation (9), we have a feasible solution for the problem on the left-hand side that has the same objective function value, establishing the relation. 
(c) This follows by combining the argument in part (b) with Lemma 3.

In the remainder of this section we will prove several tight bounds on the efficiency loss of partially optimal routing. We begin by recalling the following key results in the analysis of selfish routing, due to Roughgarden and Tardos [9].

Proposition 3 (Roughgarden-Tardos (2002) [9])

(a)

$$
\inf _{R \in \mathcal{R}^{\text {conv }}} \frac{C\left(\mathrm{x}^{S O}(R)\right)}{C\left(\mathrm{x}^{W E}(R)\right)}=0 .
$$

(a) Consider a routing instance $R=(V, A, P, s, t, X, \mathrm{l})$ where $l_{j}$ is an affine latency function for all $j \in A$. Then,

$$
\frac{C\left(\mathrm{x}^{S O}(R)\right)}{C\left(\mathrm{x}^{W E}(R)\right)} \geq \frac{3}{4} .
$$

Furthermore, the bound above is tight.

The first result shows that the worst-case efficiency loss of selfish routing is unbounded, when latency functions are only known to be convex. However, if latency functions are affine, then the proposition guarantees the tight bound on efficiency loss in part (b).

Our main theorem in this section is an extension of the results in Proposition 3 to the setting of partially optimal routing.

\section{Theorem 1}

(a)

$$
\inf _{\substack{R \in \mathcal{R}^{\text {conv }} \\ R_{0} \subset R}} \frac{C^{S O}(R)}{C^{P O R}\left(R, R_{0}\right)}=0 .
$$

(a) Consider a routing instance $R=(V, A, P, s, t, X, \mathrm{l})$ where $l_{j}$ is an affine latency function for all $j \in A$; and a subnetwork $R_{0}$ of $R$. Then:

$$
\frac{C\left(\mathrm{x}^{S O}(R)\right)}{C\left(\mathrm{x}^{P O R}\left(R, R_{0}\right)\right)} \geq \frac{3}{4} .
$$

Furthermore, the bound above is tight. 
Proof. Part (a) of the theorem is an immediate corollary of Proposition 2(a) (for $\mathcal{R}^{\prime}=$ $\mathcal{R}^{\text {conv }}$ ) and Proposition 3(b).

The remainder of the proof establishes part (b) of the theorem by proving two lemmas. The first provides a tight bound of $3 / 4$ on the ratio of the optimal routing cost to the selfish routing cost for routing instances in which the latency function of each link is a concave function. This lemma is relevant because when all latency functions are affine, the effective latency of any subnetwork under partially optimal routing is concave, as shown in the second lemma.

The proof of the following lemma uses a geometric argument that was used in [16]. This result also follows from the analysis in [10]. Here, we provide an alternative proof, which will be useful in our subsequent analysis.

Lemma 2 Let $R \in \mathcal{R}^{\text {conc }}$ be a routing instance where all latency functions are concave. Then,

$$
\frac{C\left(\mathrm{x}^{S O}(R)\right)}{C\left(\mathrm{x}^{W E}(R)\right)} \geq \frac{3}{4}
$$

Furthermore, this bound is tight.

Proof of Lemma. Consider a routing instance $R \in \mathcal{R}^{\text {conc }}$, with $R=(V, A, P, s, t, \mathrm{X}, \mathrm{l})$. Let $x^{W E}$ be the flow configuration at a Wardrop equilibrium. By Eq. (3), for all feasible solutions $\mathrm{x}$ of Problem (1), we have

$$
\begin{aligned}
C\left(\mathbf{x}^{W E}\right) & =\sum_{j \in A} x_{j}^{W E} l_{j}\left(x_{j}^{W E}\right) \\
& \leq \sum_{j \in A} x_{j} l_{j}\left(x_{j}^{W E}\right) \\
& =\sum_{j \in A} x_{j} l_{j}\left(x_{j}\right)+\sum_{j \in A} x_{j}\left(l_{j}\left(x_{j}^{W E}\right)-l_{j}\left(x_{j}\right)\right) .
\end{aligned}
$$

We next show that for all $j \in A$, and all feasible solutions $\mathbf{x}$ of Problem (1), we have

$$
x_{j}\left(l_{j}\left(x_{j}^{W E}\right)-l_{j}\left(x_{j}\right)\right) \leq \frac{1}{4} x_{j}^{W E} l_{j}\left(x_{j}^{W E}\right) .
$$

If $x_{j} \geq x_{j}^{W E}$, then since $l_{j}$ is nondecreasing, we have $l_{j}\left(x_{j}^{W E}\right) \leq l_{j}\left(x_{j}\right)$, establishing the desired relation. Assume next that $x_{j}<x_{j}^{W E}$. The term $x_{j}\left(l_{j}\left(x_{j}^{W E}\right)-l_{j}\left(x_{j}\right)\right)$ is equal to the area of the shaded rectangle in Figure 1. Consider the triangle formed by the three points

$$
\begin{aligned}
& \left(0, l_{j}\left(x_{j}^{W E}\right)\right),\left(0, l_{j}\left(x_{j}\right)-l_{j}^{\prime}\left(x_{j}\right) x_{j}\right), \\
& \quad\left(\frac{l_{j}\left(x_{j}^{W E}\right)-l_{j}\left(x_{j}\right)+l_{j}^{\prime}\left(x_{j}\right) x_{j}}{l_{j}^{\prime}\left(x_{j}\right)}, l_{j}\left(x_{j}^{W E}\right)\right) .
\end{aligned}
$$




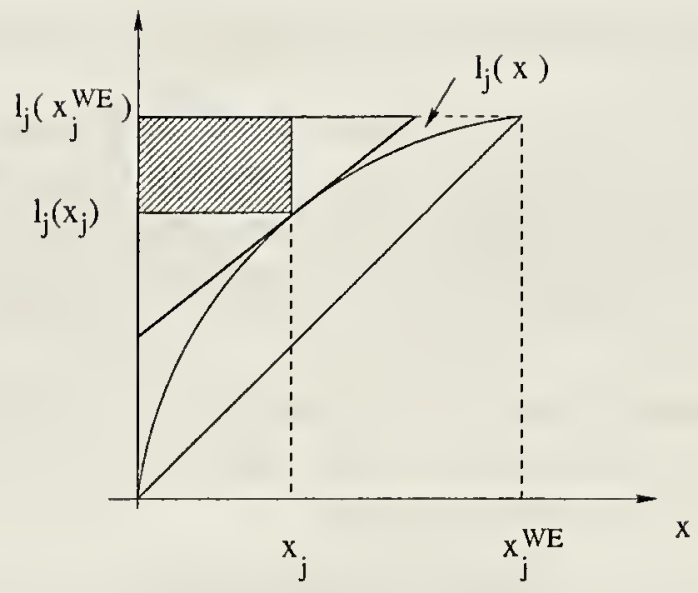

Figure 2: Illustration of the proof of Proposition 2.

Denote this triangle by $T$. It can be seen that

$$
x_{j}\left(l_{j}\left(x_{j}^{W E}\right)-l_{j}\left(x_{j}\right)\right) \leq \frac{1}{2} \operatorname{Area}(T) .
$$

By the concavity of $l_{j}$, we further have

$$
\operatorname{Area}(T) \leq \int_{0}^{x_{j}^{W E}} \int_{l_{j}(x)}^{l_{j}\left(x_{j}^{W E}\right)} d y d x \leq \frac{x_{j}^{W E} l_{j}\left(x_{j}^{W E}\right)}{2}
$$

i.e., the area of triangle $T$ is less than or equal to the area between the curves $y=l_{j}(x)$ and $y=l_{j}\left(x_{j}^{W E}\right)$ in the interval $x \in\left[0, x_{j}^{W E}\right]$, which in turn is less than or equal to half of the area $x_{j}^{W E} l_{j}\left(x_{j}^{W E}\right)$. Combining the preceding two relations, we obtain Eq. (13), which implies

$$
\sum_{j \in A} x_{j}\left(l_{j}\left(x_{j}^{W E}\right)-l_{j}\left(x_{j}\right)\right) \leq \frac{1}{4} \sum_{j \in A} x_{j}^{W E} l_{j}\left(x_{j}^{W E}\right)=\frac{1}{4} C\left(\mathrm{x}^{W E}\right) .
$$

Combining with Eq. (12), we see that for all feasible solutions $\mathbf{x}$ of Problem (1), we have

$$
\frac{3}{4} C\left(\mathrm{x}^{W E}\right) \leq \sum_{j \in A} x_{j} l_{j}\left(x_{j}\right)
$$

Since the socially optimal flow configuration $\mathrm{x}^{S O}$ is a feasible solution for Problem (1), we obtain the desired result.

The following lemma, which establishes that the effective latency $l_{0}$ of a subnetwork under partially optimal routing is concave when the latency functions are affine, completes the proof of part (b) of the theorem. 
Lemma 3 Let $R_{0}=\left(V_{0}, A_{0}, P_{0}, s_{0}, t_{0}\right)$ be a subnetwork. Assume that the latency functions of all links in the subnetwork are nonnegative affine functions, i.e., for all $j \in A_{0}$, $l_{j}\left(x_{j}\right)=a_{j} x_{j}+b_{j}$, where $a_{j} \geq 0$ and $b_{j} \geq 0$. Let $l_{0}\left(X_{0}\right)$ denote the effective latency of partially optimal routing of $X_{0}$ units of flow in the subnetwork $R_{0}$. Then $l_{0}\left(X_{0}\right)$ is a concave function of $X_{0}$.

Proof of Lemma. Since the $l_{j}$ are affine, for all $X_{0} \geq 0$, we have

$$
\begin{aligned}
l_{0}\left(X_{0}\right)=\min _{y_{p} \geq 0, p \in P} & \sum_{j \in A_{0}} \frac{a_{j} x_{j}^{2}}{X_{0}}+\frac{b_{j} x_{j}}{X_{0}} \\
\text { subject to } & \sum_{p \in P_{0}: j \in p} y_{p}=x_{j}, \quad j \in A_{0} ; \\
& \sum_{p \in P_{0}} y_{p}=X_{0} .
\end{aligned}
$$

Using the change of variables $\hat{y}_{p}=\frac{y_{p}}{X_{0}}$ for all $p \in P_{0}$, and $\hat{x}_{j}=\frac{x_{j}}{X_{0}}$ for all $j \in A_{0}$ in the preceding optimization problem, we obtain

$$
\begin{aligned}
l_{0}\left(X_{0}\right)=\min _{\hat{y}_{p} \geq 0, p \in P_{0}} & \sum_{j \in A_{0}} a_{j} X_{0} \hat{x}_{j}^{2}+b_{j} \hat{x}_{j} \\
\text { subject to } & \sum_{p \in P_{0}: j \in p} \hat{y}_{p}=\hat{x}_{j}, \quad j \in A_{0} ; \\
& \sum_{p \in P_{0}} \hat{y}_{p}=1 .
\end{aligned}
$$

Denote the feasible set of problem (14) by $Y$, i.e.,

$$
Y=\left\{\mathrm{y} \mid y_{p} \geq 0, \forall p \in P_{0}, \sum_{p \in P_{0}} y_{p}=1\right\} .
$$

Then by defining $x_{j}(\mathrm{y})=\sum_{p \in P_{0}: j \in p} y_{p}$, we can write (14) equivalently as:

$$
l_{0}\left(X_{0}\right)=\inf _{\mathbf{y} \in Y}\left[\left(\sum_{j \in A_{0}} a_{j} x_{j}(\mathrm{y})^{2}\right) X_{0}+\left(\sum_{j \in A_{0}} b_{j} x_{j}(\mathrm{y})\right)\right] .
$$

But now observe that $l_{0}\left(X_{0}\right)$ is the infimum of a collection of affine functions of $X_{0}$. By a standard result in convex analysis (see, e.g., [14], Proposition 1.2.4(c)), it follows that $l_{0}\left(X_{0}\right)$ is concave.

Combining Lemmas 2 and 3 with Proposition 2 completes the proof of part (b) of Theorem 1 . 
The preceding proof exploits the fact that the effective latency $l_{0}$ is concave in the subnetwork to establish a tight efficiency loss bound for partially optimal routing with respect to the social optimum, under the assumption that all latency functions are affine. We can apply a similar approach to develop bounds on the efficiency loss of partially optimal routing even when the latency functions may not be affine; our starting point is a result of Correa et al. [16], extending earlier work of Roughgarden [17], that gives bounds on the efficiency loss of selfish routing with general latency functions.

To state their result, we require the following definitions. Given a class of latency functions $\mathcal{L}$, we define $\beta(\mathcal{L})$ as:

$$
\beta(\mathcal{L})=\sup _{l \in \mathcal{L}, x \geq 0} \beta(l, x)
$$

with

$$
\beta(l, x)=\max _{z \geq 0} \frac{(l(x)-l(z)) z}{l(x) x},
$$

Intuitively $\beta$ is measure of the steepness of a class of latency functions; for all the cases we will consider, it is equivalent to $1-1 / \alpha(\mathcal{L})$, where $\alpha(\mathcal{L})$ is the steepness parameter defined by Roughgarden [17]. The following proposition was first proven by Roughgarden [17] for convex and differentiable latency functions, and then extended by Correa et al. to all classes of latency functions [16].

Proposition 4 Let $\mathcal{L}$ be a class of separable latency functions. Consider a routing instance $R=(V, A, P, s, t, \mathrm{X}, \mathrm{l})$ with $l_{j} \in \mathcal{L}$ for all $j \in A$. Then

$$
\frac{C\left(\mathrm{x}^{S O}(R)\right)}{C\left(\mathrm{x}^{W E}(R)\right)} \geq(1-\beta(\mathcal{L})) \text {. }
$$

Furthermore, the bound above is tight.

We emphasize that $\beta(\mathcal{L})=1 / 4$ when $\mathcal{L}$ is the class of affine latency functions, so the preceding proposition is indeed a generalization of Proposition 3.

In the spirit of Proposition 4, the following theorem generalizes the results of Theorem 1 to networks where latencies are nonnegative polynomials.

Theorem 2 Let $\mathcal{L}_{d}$ be a class of nonnegative separable polynomial latency functions of degree $d$. Consider a routing instance $R=(V, A, P, s, t, X, \mathrm{l})$ with $l_{j} \in \mathcal{L}_{d}$ for all $j \in A$, and a subnetwork $R_{0}$ of $R$. Then,

$$
\frac{C\left(\mathrm{x}^{S O}(R)\right)}{C\left(\mathrm{x}^{P O R}\left(R, R_{0}\right)\right)} \geq\left(1-\beta\left(\mathcal{L}_{d}\right)\right)
$$

where $\beta\left(\mathcal{L}_{d}\right)$ is defined in Eqs. (15)-(16). Furthermore, the bound above is tight. 
Proof. The proof proceeds as follows. First, we establish a bound on the efficiency loss of a routing instance where each latency function is the pointwise infimum of a collection of latency functions in a prespecified class. Then, we establish that if all latency functions are polynomials, the effective latency of any subnetwork under partially optimal routing is an infimum of polynomials. Combining these claims will yield the result of the proposition.

Our starting point is the following observation of Correa et al. ([16], particularly Lemma 2.3): given a class of separable latency functions $\mathcal{L}$ and a routing instance $R=(V, A, P, s, t, X, \mathrm{I})$ with $l_{j} \in \mathcal{L}$ for all $j \in A$, the following inequality holds:

$$
x_{j} l_{j}\left(x_{j}^{W E}(R)\right) \leq x_{j} l_{j}\left(x_{j}\right)+\beta(\mathcal{L}) x_{j}^{W E}(R) l_{j}\left(x_{j}^{W E}(R)\right), \forall j \in A, \forall x \geq 0 .
$$

Using this fact, we can prove the following lemma.

Lemma 4 Let $\mathcal{L}_{s}$ be a class of nonnegative separable latency functions which is closed under scaling by a constant $k \leq 1$ (i.e., for all $l \in \mathcal{L}_{s}$, we have $k l \in \mathcal{L}_{s}$ for all scalars $k \leq 1)$. Let $R=(V, A, P, s, t, \mathrm{X}, \mathrm{l})$ be a routing instance with

$$
l_{j}(x)=\inf _{z \in \mathcal{Z}_{j}}\{f(x, z)\}, \quad \forall j \in A,
$$

where: $\mathcal{Z}_{j}$ is a compact set; for each $x, f(x, \cdot)$ is a continuous function of $z$; and for each $z \in \mathcal{Z}_{j}, f(\cdot, z) \in \mathcal{L}_{s}$. Then:

$$
\frac{C\left(\mathrm{x}^{S O}(R)\right)}{C\left(\mathrm{x}^{W E}(R)\right)} \geq\left(1-\beta\left(\mathcal{L}_{s}\right)\right)
$$

Proof of Lemma. We use the bound in Eq. (17) together with a similar geometric argument used in the proof of Lemma 2 to prove the result.

Let $\mathrm{x}$ be a feasible solution of problem (1). By the definition of $l_{j}$ [cf. Eq. (18)], for all $j \in A$, there exists some $\bar{z}_{j}$ such that

$$
l_{j}\left(x_{j}\right)=f\left(x_{j}, \bar{z}_{j}\right),
$$

and

$$
l_{j}\left(x_{j}^{W E}(R)\right) \leq f\left(x_{j}^{W E}(R), \bar{z}_{j}\right) .
$$

Let $c=\frac{f\left(x_{j}^{W E}(R), \bar{z}_{j}\right)}{l_{j}\left(x_{j}^{W E}(R)\right)} \geq 1$ and define

$$
\bar{f}_{j}(y)=\frac{f\left(y, \bar{z}_{j}\right)}{c}, \quad \forall y \geq 0 .
$$

Since $c \geq 1$ and $f\left(y, \bar{z}_{j}\right) \geq 0$, it follows that $\bar{f}_{j}(y) \leq f\left(y, \bar{z}_{j}\right)$ for all $y \geq 0$, which by Eq. (19) implies that

$$
\bar{f}_{j}\left(x_{j}\right) \leq l_{j}\left(x_{j}\right) .
$$


In view of the assumption that the class $\mathcal{L}_{s}$ is closed under scaling by a constant $k \leq 1$, we have $\bar{f}_{j} \in \mathcal{L}_{s}$. Moreover, since $\bar{f}_{j}\left(x_{j}^{W E}(R)\right)=l_{j}\left(x_{j}^{W E}(R)\right)$, $\mathrm{x}^{W E}(R)$ is a Wardrop equilibrium of the routing instance $\bar{R}=(V, A, P, s, t, X, \overline{\mathrm{l}})$, where $\bar{l}_{j}=\bar{f}_{j}$ for all $j \in A$. Combining the preceding, we obtain

$$
\begin{aligned}
x_{j}\left(l_{j}\left(x_{j}^{W E}(R)\right)\right. & \left.-l_{j}\left(x_{j}\right)\right) \\
& \leq x_{j}\left(\bar{f}_{j}\left(x_{j}^{W E}(R)\right)-\bar{f}_{j}\left(x_{j}\right)\right) \\
& \leq \beta\left(\mathcal{L}_{s}\right) C\left(\mathrm{x}^{W E}(R)\right),
\end{aligned}
$$

Summing over all $j \in A$ and using an argument similar to the proof of Lemma 2 [in particular Eq. (12)], we obtain the desired result.

The following lemma characterizes effective latencies in the special case where all latency functions are nonnegative polynomials. The proof is similar to the proof of Lemma 3 , and is omitted.

Lemma 5 Let $R_{0}=\left(V_{0}, A_{0}, P_{0}, s_{0}, t_{0}\right)$ be a subnetwork. Assume that the latency functions of all links in the subnetwork are nonnegative polynomials of degree $d$, i.e., for all $j \in A_{0}, l_{j}\left(x_{j}\right)$ is a polynomial of degree $d$ such that $l_{j}\left(x_{j}\right) \geq 0$ for all $x_{j} \geq 0$. Let $l_{0}\left(X_{0}\right)$ denote the effective latency of partially optimal routing of $X_{0}$ units of flow in the subnetwork $R_{0}$. Then $l_{0}\left(X_{0}\right)$ is given by

$$
l_{0}\left(X_{0}\right)=\inf _{y \in \mathcal{Y}}\left\{f\left(X_{0}, y\right)\right\},
$$

where $\mathcal{Y}$ is a nonempty compact set, $f\left(X_{0}, y\right)$ is a continuous function of $y$, and for each $y \in \mathcal{Y}, f(\cdot, y)$ is a nonnegative polynomial of degree $d$.

Since the class of polynomial functions is closed under scaling by a constant, the preceding two lemmas immediately imply the conclusion of the theorem, as required.

The preceding theorem, together with Theorem 1, gives a tight characterization of the efficiency loss of partially optimal routing when all independently-operated subnetworks have unique entry and exit points. In particular, observe that, as long as latency functions are polynomial of bounded degree, the worst-case efficiency loss under partially optimal routing is no worse than the same worst-case value for pure selfish routing.

\section{Subnetworks with Multiple Entry and Exit Points}

We now turn to a discussion of the efficiency of partially optimal routing when independentlyoperated subnetworks can have multiple entry and exit points. Unfortunately, in this 


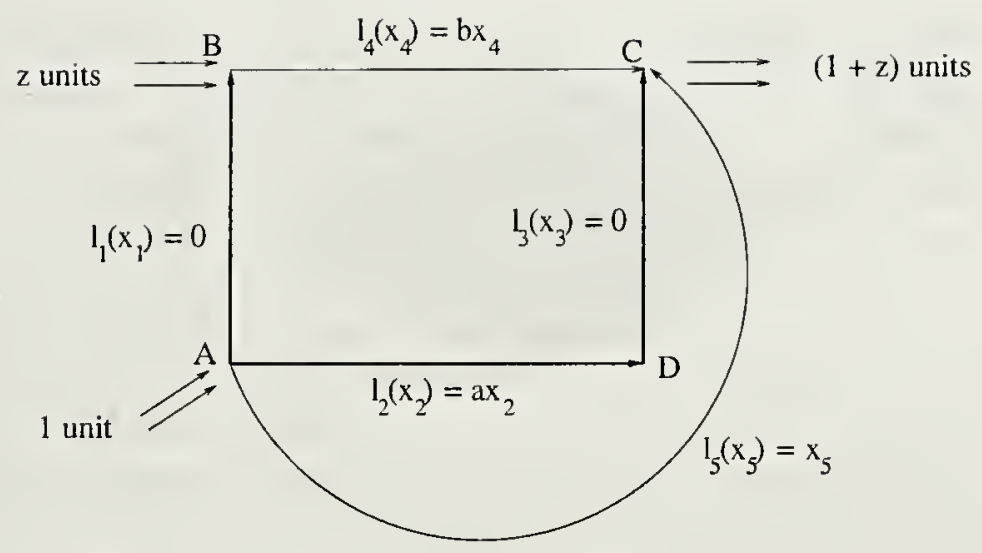

Figure 3: A subnetwork with multiple exit points.

case efficiency loss may be unbounded for even in the most restrictive case where latency functions are linear. The next example shows that the efficiency loss may be unbounded for networks that include subnetworks with multiple entry and exit points.

Example 2 Consider the general network illustrated in Figure 2. The subnetwork consists of links 1,2 , and 3 with latency functions

$$
l_{1}\left(x_{1}\right)=0, \quad l_{2}\left(x_{2}\right)=a x_{2}, \quad l_{3}\left(x_{3}\right)=0,
$$

for some $a>0$. The remaining links in the network have latency functions

$$
l_{4}\left(x_{4}\right)=b x_{4}, \quad l_{5}\left(x_{5}\right)=x_{5},
$$

for some $b>a>0$.

The link flows at the social optimum are given by:

$$
x^{S}=\left(0, \frac{1}{1+a}, \frac{1}{1+a}, z, \frac{a}{1+a}\right) .
$$

The cost of the optimal solution is

$$
C\left(x^{S}\right)=b z^{2}+\frac{a}{(1+a)^{2}}+\frac{a^{2}}{(1+a)^{2}} .
$$

For any $a>0$, the optimal routing for the subnetwork is to route all the incoming flow along link 1 . To find the POR equilibrium, let $x_{1}$ denote the amount of traffic that is routed over the subnetwork (i.e., along link 1). Assuming that $b z<1$, we solve for $x_{1}$ in the Wardrop conditions

$$
b\left(z+x_{1}\right)=\left(1-x_{1}\right) .
$$




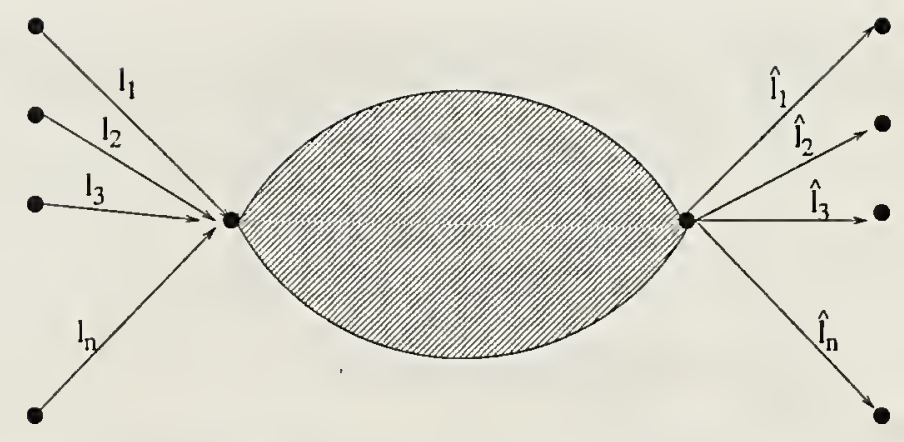

Figure 4: A special multiple entry-exit subnetwork, where the shaded area represents a single entry-exit subnetwork with an arbitrary topology.

Hence the link flows at the POR equilibrium are given by:

$$
x^{P O R}=\left(\frac{1-b z}{1+b}, 0,0, \frac{1+z}{1+b}, \frac{b+b z}{1+b}\right) .
$$

The cost of the POR equilibrium is

$$
C\left(x^{P O R}\right)=b\left(\frac{1+z}{1+b}\right)^{2}+\left(\frac{b+b z}{1+b}\right)^{2} .
$$

For a fixed $b>0$, taking the limit as $a \rightarrow 0$ and $z \rightarrow 0$, we obtain

$$
C\left(x^{S}\right) \rightarrow 0, \quad C\left(x^{P O R}\right) \rightarrow \frac{b}{1+b}>0,
$$

thus showing that the relative efficiency approaches zero.

Nevertheless, there are at least two important special cases where one can show that the efficiency loss of partially optimal routing is bounded. The first is illustrated by the network topology in Figure 4. Let $s_{1}, \ldots, s_{n}$ be $n$ entry points, and $t_{1}, \ldots, t_{n}$ be $n$ exit points. Assume that $x_{i}$ units of flow enters the network at node $s_{i}$, and is destined to node $t_{i}$, for all $i=1, \ldots, n$. The shaded area represents a single entry-exit subnetwork with an arbitrary topology.

For this structure, it is evident that the POR effective latency between nodes $s_{i}$ and $t_{i}$ is given by

$$
\tilde{l}_{i}\left(x_{i}, X\right)=l_{i}\left(x_{i}\right)+\hat{l}_{i}\left(x_{i}\right)+l_{0}(X)
$$

where $X=\sum_{i=1}^{n} x_{i}$, and $l_{0}$ is the effective latency of optimal routing within the single entry-exit subnetwork, defined as in our previous analysis. Given this structure, immediate corollaries of Theorems 1 or 2 imply that, under the assumptions of these theorems, exactly the same results apply to the model of Figure 4. 
A bound on efficiency loss can also be provided in the case when the following three conditions are satisfied: first, all latencies in the entire network are affine; second, all latencies in the subnetwork under consideration are linear (i.e., zero latency at zero flow); and third, for any given source-destination pair $(s, t)$ in the global network, there exist distinguished nodes $i_{s t}$ and $j_{s t}$ in the subnetwork, such that every path $P$ available to $(s, t)$ that passes through the subnetwork enters at $i_{s t}$ and leaves at $j_{s t}$. In this case, it is straightforward to establish that a Wardrop equilibrium in the global network leads to a Wardrop equilibrium within the subnetwork. Furthermore, the Wardrop equilibrium within the subnetwork must optimize intradomain performance. The latter claim follows because when latencies are linear, the social optimum and Wardrop equilibrium coincide (as is readily observed from the optimization problem (1)). As a result, the POR equilibrium in this setting is equivalent to the Wardrop equilibrium, and thus the efficiency loss is bounded by $3 / 4$ in this setting (cf. Proposition 3 ).

\section{Partially Optimal Routing and Subnetwork Per- formance}

In this section, we consider a model where a subnetwork chooses its routing policy to achieve the minimum (total) latency within its subnetwork. This amounts to assuming that the subnetwork ignores revenues from transmission, which is natural in this context, since we have not considered the pricing decisions of service providers (see Concluding Remarks). While optimal routing seems like the natural means to achieve this goal, end-to-end route selection may counteract any expected performance gains from this type of intradomain traffic engineering. As a result, the provider may prefer to allow traffic to route selfishly in order to reduce flow and total delay in its subnetwork. The following example illustrates this scenario.

Example 3 Consider the parallel-link network illustrated in Figure 5. The latency functions are given by

$$
l_{1}\left(x_{1}\right)=1, \quad l_{2}\left(x_{2}\right)=x_{2}^{2}, \quad l_{3}\left(x_{3}\right)=c,
$$

for some constant $c>0$. Assume that links 1 and 2 form a subnetwork, denoted by $G_{0}$, which is controlled by an independent administrator. Assume that one unit of flow is to be routed over this network.

Assume first that the flow through the subnetwork $G_{0}$ is routed selfishly, i.e., according to Wardrop equilibrium. It can be seen in this case that $\sqrt{c}$ units of traffic is routed through the subnetwork, leading to a total cost of $C\left(\mathrm{x}^{W E}\right)=c$, and a subnetwork cost of $C_{G_{0}}\left(\mathrm{x}^{W E}\right)=c \sqrt{c}$.

Assume next that the flow through the subnetwork $G_{0}$ is routed optimally, i.e., the flow is routed through the overall network according to POR equilibrium. Assume that 


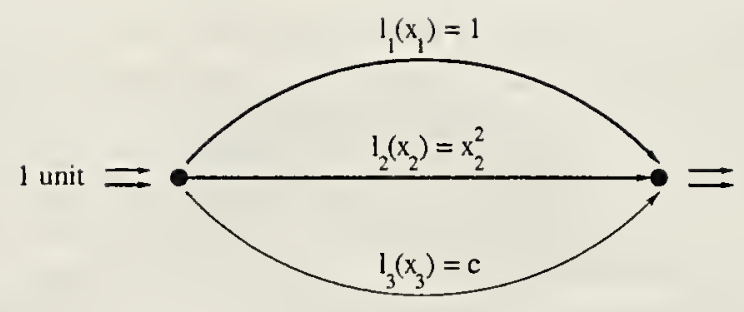

Figure 5: A parallel link network. Links 1 and 2 form a subnetwork that is controlled by an independent administrator.

the constant $c \in\left[1-\frac{2}{3 \sqrt{3}}, 1\right]$. It can be seen in this case that the entire traffic is routed through the subnetwork, leading to a total and subnetwork cost of $C\left(\mathrm{x}^{P O R}\right)=$ $C_{G_{0}}\left(\mathrm{x}^{P O R}\right)=1-\frac{2}{3 \sqrt{3}}$. Note that for $c \sqrt{c}<1-\frac{2}{3 \sqrt{3}}$, we have

$$
C_{G_{0}}\left(\mathrm{x}^{P O R}\right)>C_{G_{0}}\left(\mathrm{x}^{W E}\right) \text {. }
$$

As the preceding example demonstrates, lower-layer traffic engineering may prefer selfish to optimal routing. It is equally easy to construct examples where optimal routing will be preferred. The simplest example is a situation in which the total traffic entering the subnetwork is constant, regardless of whether selfish or optimal routing is used. This will be the case in the example above when $c>1$, and a similar analysis immediately implies that optimal routing will be preferred within the subnetwork in this case.

To gain more insights, let us next consider a "partial equilibrium" analysis of routing within a subnetwork, taking the strategies of all other subnetworks as given. To illustrate the main issues, we consider a network consisting of parallel links between a single origindestination pair with $d$ units of total traffic. Suppose that there are $N+1$ providers and each network provider owns a subset of the links in the network. We represent network provider $i$, for $i=1, \ldots, N$, by a single link with effective latency $l_{i}$ (corresponding to the intradomain routing policy chosen by provider $i$, whether optimal routing or not). We assume all these latency functions $l_{i}$ are continuous and strictly increasing.

As in the preceding discussion, we assume that if provider 0 pursues an optimal intradomain routing policy, then the effective latency is given by $l_{0}$, and if provider 0 allows purely selfish routing within his network (corresponding to a Wardrop equilibrium), the effective latency is $\tilde{l}_{0}$. To simplify the discussion here, let us also assume that $l_{0}$ and $\tilde{l}_{0}$ are both continuous and strictly increasing (this will be the case, for example, when all latency functions of the links in the subnetwork are continuous and strictly increasing). As before, recall that $\tilde{l}_{0}(x) \geq l_{0}(x)$ for all $x \geq 0$. Moreover, for simplicity, let us assume that $\tilde{l}_{0}(x)>l_{0}(x)$ if $x>0$ (though the arguments can be generalized to the case without this assumption).

We assume that the subnetwork owner can randomize between the two policies, so any convex combination of optimal and selfish routing can be achieved. In other words, 
the subnetwork owner chooses a $\delta \in[0,1]$ corresponding to an effective latency given by:

$$
m_{0}(x, \delta)=(1-\delta) l_{0}(x)+\delta \tilde{l}_{0}(x),
$$

where $\delta=0$ corresponds to optimal routing, while $\delta=1$ corresponds to selfish routing.

We continue to use $\mathrm{x}^{P O R}$ to denote a Wardrop equilibrium with respect to the latency functions $m_{0}, l_{1}, \ldots, l_{N}$, so that $\mathrm{x}^{P O R}$ satisfies:

$$
\begin{aligned}
m_{0}\left(x_{0}^{P O R}, \delta\right) & \geq \lambda \\
l_{i}\left(x_{i}^{P O R}\right) & \geq \lambda \text { for } i=1, \ldots, N \\
\sum_{i=0}^{N} x_{i}^{P O R} & =d \\
x_{i}^{P O R} & \geq 0 \text { for } i=0, \ldots, N \\
\lambda & =\min \left\{m_{0}\left(x_{0}^{P O R}, \delta\right), l_{1}\left(x_{1}^{P O R}\right), \ldots, l_{N}\left(x_{N}^{P O R}\right)\right\} .
\end{aligned}
$$

First consider the routing of flow through the links $1, \ldots, N$. If a total flow $x$ is routed through links $1, \ldots, N$, then the resulting flow allocation must satisfy:

$$
\begin{aligned}
l_{i}\left(x_{i}\right) & =\min \left\{l_{1}\left(x_{1}\right), \ldots, l_{N}\left(x_{N}\right)\right\} \text { if } x_{i}>0 \\
\sum_{i=1}^{N} x_{i} & =x \\
x_{i} & \geq 0, i=1, \ldots, N
\end{aligned}
$$

In view of the assumption that $l_{1}, \ldots, l_{N}$ are strictly increasing, the preceding equations have a unique solution. We define $l_{R}(x)$ as the latency at this solution, i.e.,

$$
l_{R}(x)=\min \left\{l_{1}\left(x_{1}\right), \ldots, l_{N}\left(x_{N}\right)\right\},
$$

where $\left(x_{1}, \ldots, x_{N}\right)$ is the unique solution to (21)-(23). Since each $l_{i}$ is strictly increasing and continuous, the function $l_{R}$ is also strictly increasing and continuous.

Next consider the traffic engineering problem faced by subnetwork 0 . The network provider will choose a value of $\delta$ that minimizes the total latency inside the subnetwork, given that traffic will follow the Wardrop equilibrium pattern for the resulting effective latencies. Formally, the optimization problem of subnetwork 0 is the following:

$$
\min _{0 \leq x_{0} \leq d, \delta \in[0,1]}\left((1-\delta) l_{0}\left(x_{0}\right)+\delta \tilde{l}_{0}\left(x_{0}\right)\right) x_{0}
$$

subject to

$$
\begin{aligned}
(1-\delta) l_{0}(0)+\delta \tilde{l}_{0}(0) & \geq l_{R}(d), \quad \text { if } x_{0}=0 \\
(1-\delta) l_{0}(d)+\delta \tilde{l}_{0}(d) & \leq l_{R}(0), \quad \text { if } x_{0}=d \\
(1-\delta) l_{0}\left(x_{0}\right)+\delta \tilde{l}_{0}\left(x_{0}\right) & =l_{R}\left(d-x_{0}\right), \quad \text { if } 0<x_{0}<d
\end{aligned}
$$


Since $\tilde{l}_{0}\left(x_{0}\right) \geq l_{0}\left(x_{0}\right)$ for all $x_{0} \geq 0$, and $l_{R}$ is strictly increasing, as $\delta$ increases from $\delta=0$ (purely optimal routing) to $\delta=1$ (purely selfish routing), the flow routed through subnetwork 0 at the POR equilibrium must be nonincreasing.

Next note that when $\tilde{l}_{0}(0) \geq l_{R}(d)$, the subnetwork can achieve the minimum total latency of zero by choosing $\delta=1$ (since the POR equilibrium will route no traffic across subnetwork 0 ). Similarly, if $\tilde{l}_{0}(d) \leq l_{R}(0)$, then regardless of provider 0 's policy, all the flow will be routed across subnetwork 0 . As a result, in this scenario the optimal strategy is $\delta=0$ (optimal routing), as this minimizes the total latency. For the remainder of this section, we assume that $\tilde{l}_{0}(0)<l_{R}(d)$ and $\tilde{l}_{0}(d)>l_{R}(0)$. Since $\tilde{l}_{0} \geq l_{0}$, this also implies

$$
l_{0}(0) \leq \tilde{l}_{0}(0)<l_{R}(d) \text {. }
$$

We now proceed to define the maximum and minimum flow that will flow through subnetwork 0 over all possible choices of routing policy. The condition (25), together with the fact that $\tilde{l}_{0}$ and $l_{R}$ are strictly increasing and continuous, ensures that the following equation has a unique solution $x_{0}^{M I N}>0$ :

$$
\tilde{l}_{0}\left(x_{0}^{M I N}\right)=l_{R}\left(d-x_{0}^{M I N}\right) .
$$

Moreover, given our assumptions, $x_{0}^{M I N}$ is the minimum flow that can go through subnetwork 0 (achieved exactly when $\delta=1$, i.e., at purely selfish routing).

The maximum possible-flow through subnetwork 0 will depend on the relative values of $l_{0}(d)$ and $l_{R}(0)$. Formally, we define $x_{0}^{M A X}$ as follows. If $l_{0}(d) \leq l_{R}(0)$, then we let $x_{0}^{M A X}=d$, since choosing $\delta=0$ (optimal routing) will lead to all traffic flowing through subnetwork 0 . On the other hand, if $l_{0}(d)>l_{R}(0)$, we let $x_{0}^{M A X}$ be the unique solution to the following equation:

$$
l_{0}\left(x_{0}^{M A X}\right)=l_{R}\left(d-x_{0}^{M A X}\right), \quad \text { if } l_{0}(d)>l_{R}(0) .
$$

With these definitions, $x_{0}^{M A X}$ is the maximum flow for subnetwork 0 (achieved exactly when $\delta=0$, i.e., at optimal routing). We define $c_{0}\left(x_{0}^{M A X}\right)$ as the cost to the owner of subnetwork 0 at this optimal routing; i.e.,

$$
c_{0}\left(x_{0}^{M A X}\right)= \begin{cases}d l_{0}(d), & \text { if } l_{0}(d) \leq l_{R}(0) \\ x_{0}^{M A X} l_{R}\left(d-x_{0}^{M A X}\right), & \text { if } l_{0}(d)>l_{R}(0) .\end{cases}
$$

Clearly, any flow $x_{0} \in\left[x_{0}^{M I N}, x_{0}^{M A X}\right]$ is achievable. To achieve a flow $x_{0} \in\left[x_{0}^{M I N}, x_{0}^{M A X}\right)$, the owner of subnetwork should choose $\delta$ such that:

$$
\delta=\frac{l_{R}\left(d-x_{0}\right)-l_{0}\left(x_{0}\right)}{\tilde{l}_{0}\left(x_{0}\right)-l_{0}\left(x_{0}\right)},
$$

where $0 \leq \delta \leq 1$ since: (1) the definition of $x_{0}^{M A X}$ ensures that $l_{R}\left(d-x_{0}\right)>l_{0}\left(x_{0}\right)$; and (2) $\tilde{l}_{0}\left(x_{0}\right)>l_{0}\left(x_{0}\right)$ for all $x_{0}>0$. Finally, using the definition of $\delta$ in (26), observe 
that for all $x_{0} \in\left[x_{0}^{M I N}, x_{0}^{M A X}\right)$, we have the relation $m_{0}\left(x_{0}, \delta\right)=l_{R}\left(d-x_{0}\right)$. In other words, if the subnetwork owner chooses policy $\delta$ according to (26), the flow through the subnetwork will be $x_{0}$, and the resulting latency will be $l_{R}\left(d-x_{0}\right)$.

As a result, the optimization problem for the owner of subnetwork 0 becomes:

$$
\min \left\{\min _{x_{0} \in\left[x_{0}^{M I N}, x_{0}^{M A X}\right)}\left[x_{0} l_{R}\left(d-x_{0}\right)\right], c_{0}\left(x_{0}^{M A X}\right)\right\},
$$

the solution of which determines the delay-minimizing routing policy of the subnetwork. If the solution yields $x_{0} \in\left[x_{0}^{M I N}, x_{0}^{M A X}\right)$, the subnetwork owner should choose $\delta$ in accordance with (26). If the solution yields $x_{0}=x_{0}^{M A X}$, the subnetwork owner should choose $\delta=0$ (pure optimal routing). If the game between service providers is one of complete information, all the latency functions are common knowledge and the owner of the subnetwork can compute $x_{0}^{M I N}, x_{0}^{M A X}$, and $l_{R}$, and hence the optimal flow through the subnetwork. If we assume that $l_{0}(d)>l_{R}(0)$, we can intuitively understand the solution: If $x_{0} l_{R}\left(d-x_{0}\right)$ increases as $x_{0}$ increases in the neighborhood of $x_{0}^{M I N}$, the provider will (locally) prefer selfish routing. Similarly, if $x_{0} l_{R}\left(d-x_{0}\right)$ decreases as $x_{0}$ decreases in the neighborhood of $x_{0}^{M A X}$, the provider prefers selfish routing.

This analysis shows that with complete information and a parallel-link network, the delay-minimizing policy of the network is straightforward to characterize. We leave several issues for future work: the analysis of networks with more general topologies; general equilibrium structures where all subnetworks optimize; situations in which latency functions of other providers are unknown; and scenarios in which the objective of subnetworks may be profit maximization rather than delay minimization.

\section{Concluding Remarks}

A newly-emerging paradigm in the analysis of large-scale communication networks recognizes and quantifies the inefficiencies resulting from the selfish behavior of flows within the network, which choose the minimum delay routes, ignoring the congestion externalities they create on the overall network (e.g., $[9,10,11])$. Even though selfish routing, where transmission follows the least cost path for each source-destination pair, is a reasonable approximation for routing across domains within large-scale networks, service providers operating their own administrative domains typically redirect traffic within their own networks to achieve better performance (see, e.g., $[2,3,4,5,6])$. This overlay routing structure of large-scale communication networks, particularly the Internet, has not previously been modeled or analyzed.

This paper provides a model of partially optimal routing that captures these essential features. While source-destination pairs transmit flows across the least cost paths, service providers controlling the subnetworks that make up the large-scale global network use traffic engineering to reduce delay within their own administrative domains. Endusers perceive the delays resulting from the traffic engineering of the network providers. 
We formulate and analyze the equilibria of this global network with partially optimal routing.

Even though traffic engineering within parts of the overall network may be conjectured to reduce congestion externalities and improve overall network performance, we show this not to be the case. In particular, if the global network exhibits the Braess' paradox, traffic engineering that reduces delays within a subnetwork may worsen the performance of the overall network. More specifically, we prove that if partially optimal routing leads to an increase in overall delay relative to selfish routing over all links, Braess' paradox must occur in the global network.

Much of the paper quantifies the potential inefficiency of partially optimal routing relative to the system optimum in the case where all independently-operated subnetworks have single entry-exit points and delays can be modeled by latency functions of a specific class, such as affine or nonnegative polynomials of bounded degree. For example, with affine latency functions, we establish that the performance of partially optimal routing is no worse than $25 \%$ relative to the system optimum.

In contrast to these results that match the corresponding bounds for selfish routing throughout the whole network, when subnetworks have multiple entry-exit points, the performance of partially optimal routing can be arbitrarily bad, even with linear latencies. This result suggests that special care needs to be taken in the regulation of traffic in large-scale networks overlaying selfish source routing together with traffic engineering within subnetworks.

We also provide conditions for service providers to prefer to engage in traffic engineering rather than allowing all traffic to route selfishly within their network. The latter is a possibility because selfish routing may discourage entry of further traffic into their subnetwork, reducing total delays within the subnetwork, which may be desirable for the network provider when there are no prices per unit of transmission.

We believe that the model of partially optimal routing presented in this paper is a good approximation to the functioning of large-scale communication networks, such as the Internet, and raises a number of interesting questions for further investigation. Possible areas of further study include:

1. Quantification of the loss of efficiency of partially optimal routing relative to selfish routing throughout the entire network.

2. Analysis of dynamics and stability of partially optimal routing in large-scale communication networks.

3. Analysis of simple regulation schemes that can prevent realization of worst-case performance losses in networks with partially optimal routing.

4. Quantification of the loss of efficiency of partially optimal routing relative to the system optimum in specific network topologies incorporating subnetworks with multiple entry-exit points and multicommodity flows. 
5. Analysis of the equilibrium of routing patterns when multiple service providers simultaneously and strategically decide the extent of traffic engineering.

6. Analysis of partially optimal routing when service providers do not simply minimize total delay, but charge for transmission through their networks and maximize profits, taking into account the impact of delays within their network on their revenues.

\section{References}

[1] J. Rexford, Route optimization in IP networks. Springer Science and Business Media, 2006, pp. 679-700.

[2] L. Qiu, Y. Yang, Y. Zhang, and S. Shenker, "On selfish routing in internet-like environments," in Proceedings of ACM SIGCOMM, 2003, pp. 151-162.

[3] H. Zhang, Y. Liu, W. Gong, and D. Towsley, "Understanding the interaction between overlay routing and traffic engineering," University of Massachusetts CMPSCI, Technical Report 04-63, 2004.

[4] — , "On the interaction between overlay routing and underlay routing," in Proceedings of IEEE Infocom, 2005, pp. 2543-2553.

[5] H. Han, S. Shakkottai, C. Hollot, R. Srikant, and D. Towsley, "Overlay TCP for multi-path routing and congestion control," 2004, submitted.

[6] F. P. Kelly and T. Voice, "Stability of end-to-end algorithms for joint routing and rate control," Computer Communication Review, vol. 35, no. 2, pp. 5-12, 2005.

[7] M. Beckmann, C. B. McGuire, and C. B. Winsten, Studies in the Economics of Transportation. New Haven, Connecticut: Yale University Press, 1956.

[8] A. Pigou, The Economics of Welfare. London, U.K.: Macmillan, 1920.

[9] T. Roughgarden and É.. Tardos, "How bad is selfish routing?" Journal of the ACM, vol. 49, no. 2, pp. 236-259, 2002.

[10] J. Correa, A. Schulz, and N. Stier-Moses, "Selfish routing in capacitated networks," Mathematics of Operations Research, vol. 29, no. 4, pp. 961-976, 2002.

[11] F. P. Kelly, "Network routing," Philosophical Transactions: Physical Sciences and Engineering, vol. 337, no. 1647, pp. 343-367, 1991. 
[12] S. Dafermos and F. Sparrow, "The traffic assignment problem for a general network," Journal of Research of the National Bureau of Standards-B. Mathematical Sciences, vol. 73, no. 2, pp. 91-118, 1969.

[13] M. Smith, "The existence, uniqueness and stability of traffic equilibria," Transportation Research, vol. 13B, pp. 295-304, 1979.

[14] D. Bertsekas, A. Nedic, and A. Ozdaglar, Convex Analysis and Optimization. Cambridge, Massachusetts: Athena Scientific, 2003.

[15] I. Milchtaich, "Network topology and the efficiency of equilibrium," Department of Economics, Bar-Ilan University, Working Paper 12-01, 2005.

[16] J. Correa, A. Schulz, and N. Stier-Moses, "On the inefficiency of equilibria in congestion games," in Proceedings of the 11th Conference on Integer Programming and Combinatorial Optimization, vol. 3509, 2005, pp. 167-181.

[17] T. Roughgarden, "The price of anarchy is independent of the network topology," in Proceedings of ACM Symposium on the Theory of Computing, 2002, pp. 428-437. 



$=5$,

45)

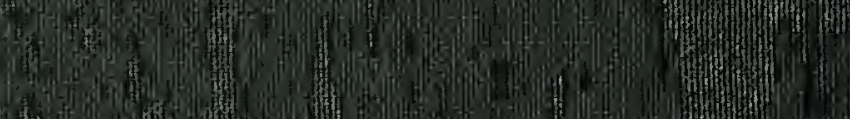

(1)

(19)

(2)

mons

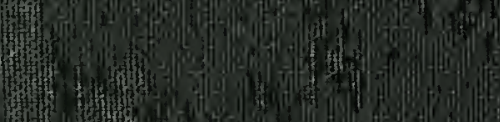

H.

Thi

7.

Hin

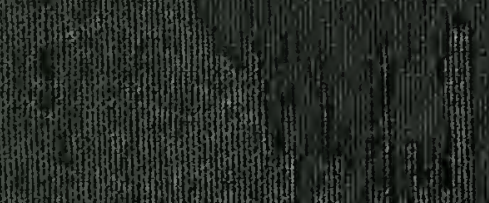

(3)

4.

matm

3.

(13)

7.

H.

A

6.

4.

-

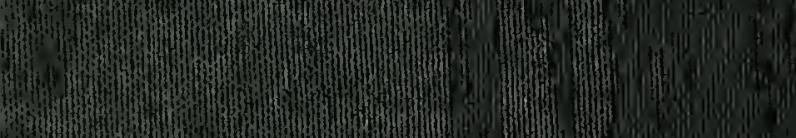
A

b.

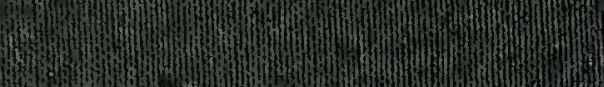

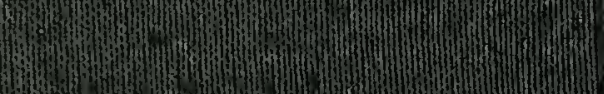

10.

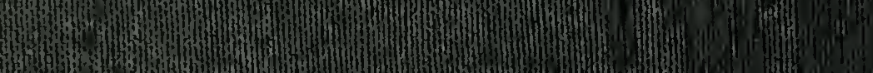
(-m)

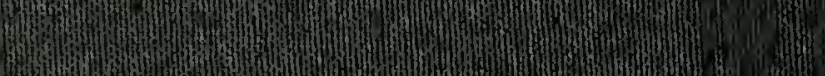

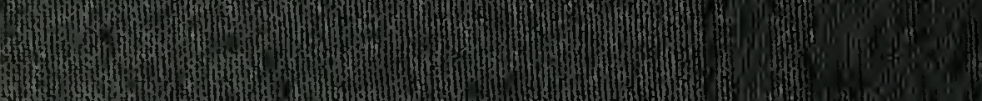

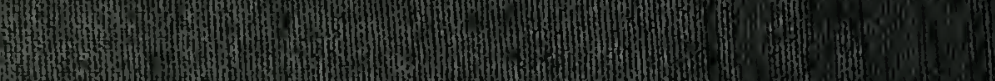

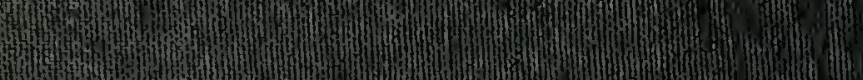
mam $\mathrm{H}_{\mathrm{m}} \mathrm{m}$

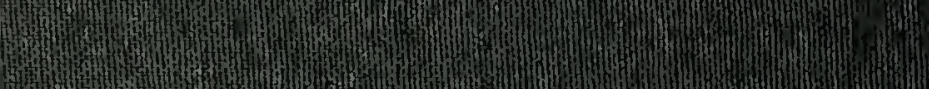

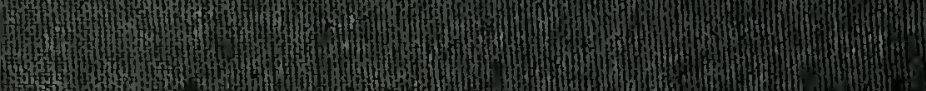

